\title{
An Assessment of Epithelial and Mesenchymal Phenotypes in Experimental and Clinical Pulmonary Fibrosis
}

\author{
Rebecca Dunmore, ${ }^{1}$ Alan M. Carruthers, ${ }^{1}$ Matthew J. Bell, ${ }^{1}$ Huilan Zhang, ${ }^{2}$ \\ Cory M. Hogaboam, ${ }^{2}$ Xueyang Peng, ${ }^{3}$ Erica L. Herzog, ${ }^{3}$ Darryl A. Knight,, 5 \\ Fernando J. Martinez, ${ }^{6}$ Matthew A. Sleeman, ${ }^{1}$ and Lynne A. Murray ${ }^{1}$ \\ ${ }^{1}$ Department of Respiratory, Inflammation and Autoimmunity, MedImmune Ltd., Granta Park, Cambridge CB21 6GH, UK \\ ${ }^{2}$ Department of Pathology, University of Michigan Medical School, Ann Arbor, MI 48109, USA \\ ${ }^{3}$ Department of Internal Medicine, Yale University School of Medicine, 300 Cedar Street, TAC 441S, New Haven, CT 06520, USA \\ ${ }^{4}$ James Hogg iCAPTURE Centre, Institute for Heart and Lung Health, Vancouver, BC, Canada V6Z 1 Y6 \\ ${ }^{5}$ Department of Anesthesiology, Pharmacology and Therapeutics, University of British Columbia, Vancouver, BC, Canada V6Z 1 Y6 \\ ${ }^{6}$ Division of Pulmonary and Critical Care Medicine, Department of Internal Medicine, University of Michigan Health System, \\ Ann Arbor, MI 48109, USA
}

Correspondence should be addressed to Lynne A. Murray, murrayl@medimmune.com

Received 11 June 2012; Accepted 12 July 2012

Academic Editors: J. Ho and A. Miyazato

Copyright (C 2012 Rebecca Dunmore et al. This is an open access article distributed under the Creative Commons Attribution License, which permits unrestricted use, distribution, and reproduction in any medium, provided the original work is properly cited.

Epithelial injury has been implicated as a driving factor for the pathogenesis of idiopathic pulmonary fibrosis (IPF). In this study we investigated changes in epithelial and mesenchymal markers in experimental models of fibrosis and associated this with IPF. TGF $\beta_{1}$ induced an epithelial to mesenchymal transition (EMT) phenotype in A549 cells and normal human bronchial epithelial cells, with A549 cells exhibiting a more profound transition to a mesenchymal phenotype. TGF $\beta_{1}$ overexpression in the lungs of mice resulted in an early increase in mesenchymal cell markers and apoptotic genes that preceded collagen deposition, suggesting an early epithelial injury triggers the downstream fibrotic response. In contrast, bleomycin had a gradual increase in mesenchymal cell marker and a decrease in E-cadherin expression that correlated with collagen protein deposition. Finally, we compared normal healthy lung tissue with surgical lung biopsies from IPF patients and observed alterations in epithelial and mesenchymal cell markers, as well as an increase in the apoptotic marker GSK3 $\beta$. Interestingly, the mesenchymal changes were more profound in rapidly progressive patients in comparison to IPF patients with slowing progressing disease. In summary, this study provides evidence of alterations in epithelial and mesenchymal markers in experimental models of lung fibrosis and how these findings are relevant to clinical disease.

\section{Introduction}

Idiopathic pulmonary fibrosis (IPF) is a chronic progressive disease of the lung with unknown aetiology and a median survival time is 2.8 years from diagnosis [1]. In the UK alone, the incidence of IPF has more than doubled in the past 15 years and there are around 5000 new cases of IPF identified each year [2]. There is currently no effective treatment for the disease, aside from lung transplantation, which improves clinical outcome [3].

Treatment of IPF patients with anti-inflammatory drugs has had little desired effect on the disease, suggesting that the pathogenesis of IPF is yet to be fully explained. It is now more widely accepted that inflammation is not the primary feature of IPF and that recurrent epithelial injury and aberrant wound healing is the main mechanism of this disease [4-7].

Alveolar type II cell injury is an early feature in IPF [8], with increased mediators of alveolar cell apoptosis in IPF lung biopsies and bronchoalveolar lavage being observed [9]. Moreover, phenotypic changes in epithelial cells have been reported in the lungs of IPF patients, whereby epithelial cells express mesenchymal markers concomitantly [10]. Due to this immunohistochemical phenomenon, it has been 
hypothesized that epithelial to mesenchymal transition (EMT) is one pathway by which fibroblasts accumulate in the lungs of IPF patients [10-12].

TGF $\beta_{1}$ is a potent inducer of EMT in multiple organs, including the lung [12] and it has recently been reported that EMT could be used as a potential prognostic indicator in IPF [13]. Extensive in vitro work has shown that epithelial cells treated with TGF $\beta_{1}$ will begin the process of EMT through increased expression of mesenchymal markers, basement membrane destruction, and finally cell detachment, yielding a mesenchymal phenotype $[14,15]$. TGF $\beta_{1}$ is aberrantly expressed in the epithelial cells in the lungs of IPF patients and found to be increased in BAL samples from patients $[16,17]$. Therefore it is plausible that local TGF $\beta_{1}$ levels could be enhancing an EMT-like phenotype in the lungs of IPF patients.

To date, evidence that EMT exists in disease-relevant animal models and IPF patients is limited and controversial. Here, using the bleomycin-induced lung fibrosis and a TGF $\beta_{1}$ transgenic mouse model, we correlate the changes in epithelial and mesenchymal markers and markers of apoptosis, with the extent of fibrosis. We then translated these findings into clinical setting by assessing the expression levels of epithelial and mesenchymal markers in lung biopsies from patients with slowly progressing and rapidly progressing forms of IPF.

\section{Materials and Methods}

2.1. Materials. TGF $\beta_{1}$ (R\&D Systems, Abingdon, UK), antiE-cadherin (R\&D Systems Abingdon, UK), anti-fibronectin (R\&D Systems, Abingdon, UK), anti-fibronectin-EDAFITC (Santa Cruz, Heidelberg, Germany), anti-vimentinTRITC (Santa Cruz, Heidelberg, Germany), anti-CollagenI (Abcam, Cambridge, UK), secondary anti-rabbit-Alex 888 (Invitrogen, Paisley, UK), $\beta$-tubulin (LI-COR), Hoeschtt 33358 (Invitrogen, Paisley, UK).

2.2. Cell Culture. Human type II alveolar epithelial cells (A549) supplied from the ATCC were maintained in F12K Hams (Invitrogen, Paisley, UK) supplemented with 10\% foetal bovine serum (SAFC Biosciences, Andover, UK), penicillin $(100 \mathrm{U} / \mathrm{mL})$ (Invitrogen, Paisley, UK), and streptomycin $(100 \mu \mathrm{g} / \mathrm{ml})$ (Invitrogen, Paisley, UK). Normal human bronchial epithelial (NHBE, nonsmoker, donor 105116) cells supplied by Lonza were maintained in BEGM (bronchial epithelial cell growth medium) BulletKit (Lonza). Both cell types were cultured at $37^{\circ} \mathrm{C}$ in the presence of $5 \% \mathrm{CO}_{2}$.

2.3. EMT Assay. A549 cells were seeded into 96-well flat bottom plates at $1.5 \times 10^{4}$ cells/well for QuantiGene 2.0 branched DNA assay and $0.5 \times 10^{4}$ cells/well for morphology and immunocytochemistry. NHBE cells were seeded into 96well flat bottom plates or 12 -well plates at $3 \times 10^{4}$ cells/well or $12 \times 10^{4}$ cells/well, respectively, for QuantiGene 2.0 branched DNA assay or into 12 -well plates at $12 \times 10^{4}$ cells/well for protein analysis via western blotting. Prior to stimulation cells A549 cells were maintained in medium with reduced FBS (1\%) and NHBEs cells were maintained in bronchial epithelial cell basal medium (BEBM) (Lonza) supplemented with retinoic acid, insulin, and antibiotics (Lonza) only, overnight. TGF $\beta_{1}$ was added to the cells and left to incubate for 24 hours for mRNA analysis and 48-72 hours for protein analysis.

2.4. mRNA Analysis. For in vitro samples, branched DNA technology using QuantiGene 2.0 reagent system (Affymetrix) was used to quantify gene expression levels according to manufacturer's guidelines. Transcript levels were normalised to PPIB and fold change compared to controls was calculated. For gene analysis from lung tissue, total RNA was obtained using either TRIzol reagent (Invitrogen, Paisley, UK) or RNeasy fibrous tissue minikit (QIAGEN) according to the manufacturer's instructions. RNA was reversetranscribed into cDNA using the high-capacity cDNA kit (Applied Biosystems) and gene expression levels were determined using qRT-PCR and analysed using an ABI Prism 7900HT sequence detector (Applied Biosystems). Transcript levels were normalized to $18 \mathrm{~s}$ and fold change was calculated compared to matched animal controls using $2^{-\Delta \Delta C t}$.

2.5. Nu-PAGE and Western Blotting. Cells were scraped and lysed in RIPA buffer (Sigma-Aldrich, Poole, UK) containing protease inhibitors (Roche Diagnostics, East Sussex, UK). Cell suspensions were cleared by centrifugation at $10000 \mathrm{rpm}$ for 10 minutes at $4^{\circ} \mathrm{C}$. Protein was loaded onto Bis-Tris (4-12\%) Nu-PAGE gels followed by electrophoresis and then transferred to PVDF membranes using the iBlot dry blotting system (Invitrogen). Membranes were blocked in Odyssey blocking buffer (LI-COR) for 2 hours and then probed with appropriate primary antibodies in Odyssey blocking buffer supplemented with $0.02 \%$ Tween20 overnight at $4^{\circ} \mathrm{C}$. Membranes were washed 4 times in PBS-T for 5 minutes each and were then probed with the appropriate IRDye $800 \mathrm{cw}$ conjugated secondary antibody (LI-COR) in Odyssey blocking buffer supplemented with $0.02 \%$ Tween-20. Membranes were washed as before and left to dry before scanning on the Odyssey Clx Infrared Imaging System (LI-COR).

2.6. Immunocytochemistry. All immunocytochemistry was performed in 96-well plates at room temperature. Following treatment with TGF $\beta_{1}$ or control for 48 hours, medium was removed and cells were fixed in $100 \mu \mathrm{L}$ of $4 \%$ formaldehyde (Sigma-Aldrich, Poole, UK) for 20 minutes. Cells were then washed three times in D-PBS $(200 \mu \mathrm{L})$. Cells were then permeabilised with $100 \mu \mathrm{L}$ of $0.2 \%$ Triton X-100 in D-PBS for 10 minutes and then rinsed 3 times in D-PBS. Following this $100 \mu \mathrm{L}$ of blocking buffer (D-PBS/1\% BSA/2\% FBS) was added to the cells for 30 minutes. Cells were then probed with the appropriate conjugated primary antibody and nuclear stain (Hoechst 33358), diluted in blocking buffer, overnight at $4^{\circ} \mathrm{C}$. Cells were washed 3 times in D-PBS and stored in $200 \mu \mathrm{L}$ of PBS. Cells were visualised on an ArrayScan (Cellomics). 
2.7. TGF $\beta_{1}$ Transgenic Mouse Model. All TGF $\beta_{1}$ transgenic mouse experiments were approved by the Yale School of Medicine Institutional Animal Care and Use Committee in accordance with federal guidelines. The CC10-tTS-rtTA$\mathrm{TGF} \beta_{1}$ transgenic mice used in this study have been described previously [18]. These mice use the Clara cell 10$\mathrm{kD}$ protein $(\mathrm{CC} 10)$ promoter to specifically target bioactive human TGF $\beta_{1}$ to the lung. These mice were backcrossed for more than 10 generations onto a C57BL/6 background, bred and genotyped as previously described [18]. 6-week-old Transgenic $^{+}$mice and Transgenic ${ }^{-}$littermate controls were randomized to normal water or water containing $0.5 \mathrm{mg} / \mathrm{mL}$ doxycycline as described previously [18].

2.8. Bleomycin-Induced Model of IPF. Six-to-eight-week-old female C57BL/6 mice (Charles River, Margate, UK) weighing $18-21 \mathrm{~g}$ were anaesthetised with ketamine $(80 \mathrm{mg} / \mathrm{kg})$ and xylazine $(8 \mathrm{mg} / \mathrm{kg})$ i.p. Bleomycin sulphate $(0.015 \mathrm{U} /$ $30 \mu \mathrm{L} / \mathrm{mouse}$, Sigma, Poole, UK) was administered as previously described [19].

2.9. Total Lung Collagen Assays. Lungs from the TGF $\beta_{1}$ transgenic mice were homogenized in antiprotease buffer (Roche Diagnostics Corp., Indianapolis, Indiana, USA) and processed as previously described [20]. Total soluble collagen levels in the TGFb Tg model were measured by Sircol Assay (BioColor, Ireland) according to the manufacturer's instructions. Lungs from the bleomycin model were assessed for hydroxyproline levels as previously described [19].

2.10. IPF Lung Samples. All surgical lung biopsies (SLBs) were obtained from the patient at the time of diagnosis of IPF. Patients were diagnosed with IPF using a multidisciplinary approach involving clinicians, radiologists, and pathologists [21]. A University of Michigan Institutional Review Board approved this study, and written informed consent was obtained from each IPF patient. IPF patients were retrospectively grouped into a rapidly progressive group $(n=9$; 2 females and 7 males; median age 66$)$ based on the following criteria during the first year of followup: mortality or acute exacerbation, percent forced vital lung capacity (FVC) decrease of $\geq 10 \%$, and percent diffusing capacity of carbon monoxide $\left(\mathrm{DL}_{\mathrm{CO}}\right.$ ) decrease of $\geq 15 \%$. IPF patients that did not meet these criteria over the first year of followup were clinically diagnosed as slowly progressive $(n=6 ; 4$ females and 2 males; median age $=61$ years). Samples taken from disease-free patient autopsies were used as normal controls $(n=10 ; 5$ females and 5 males; median age $=24$ years). A University of British Columbia Institutional Review Board approved the procurement and analysis of the healthy control lung tissues.

2.11. Statistical Analysis. The data show the mean \pm SEM of at least three independent experiments $(n=3)$ unless otherwise indicated. Statistical significance was determined using Student's $t$-test or one-way ANOVA with Dunnett's posttest where appropriate. $P$ values less than 0.05 were considered statistically significant.

\section{Results}

3.1. TGF $\beta_{1}$ Induces EMT In Vitro. EMT is a common feature of tumour development. Here we initially used a lung epithelial tumour cell line, A549, which is commonly used in EMT assays [22-24], to profile the TGF $\beta_{1}$-induced changes in EMT markers. A549 cells were treated with TGF $\beta_{1}$ for 24 hours and a panel of EMT associated genes were measured. The epithelial markers E-cadherin and SIP-1 both showed a downregulation following the addition of TGF $\beta_{1}$ in a dose-dependent manner (Figure 1(a)). In contrast, there was an upregulation of mesenchymal markers, also in a dosedependent manner, including $\mathrm{N}$-cadherin, vimentin, and collagen Ia1 (Figure 1(b)). Treating A549 cells with TGF $\beta_{1}$ for 72 hours also induced morphological changes to the cells. As shown in the phase contrast images (Figure 1(c)), the cells changed from a typical epithelial cobblestone appearance (untreated) to a spindle-shaped morphology, characteristic of a mesenchymal cell, in response to TGF $\beta_{1}$. This change in cell morphology was also associated with increased mesenchymal protein expression, as shown by immunofluorescent staining for fibronectin-EDA, vimentin, and collagen I (Figure 1(d), all shown in red, Hoeschtt 33358 nuclear stain is shown in blue).

Since A549 cells are lung carcinoma derived, we then assessed whether primary human bronchial epithelial (NHBE) cells would also undergo EMT in response to TGF $\beta_{1}$. Treating NHBE cells with TGF $\beta_{1}(1$ and $10 \mathrm{ng} / \mathrm{mL}$ ) for 24 hours resulted in a downregulation of the epithelial marker E-cadherin (Figure 2(a)) and a concomitant upregulation of the mesenchymal markers vimentin, collagen Ia1, and fibronectin-1 (Figure 2(b)). Following a 48-hour treatment with TGF $\beta_{1}(10 \mathrm{ng} / \mathrm{mL}$, "+"), western blot analysis (Figure 2(c)) was performed and confirmed an increase in fibronectin-1 in response to TGF $\beta_{1}$; however, the epithelial marker E-cadherin appeared unchanged under these conditions. Moreover, it was also noted that NHBE cells showed little change in morphology when treated with TGF $\beta_{1}$ and appeared to maintain an epithelial-like phenotype (data not shown). This may account for the minimal changes in Ecadherin at protein level as junctional proteins would still be intact to maintain this epithelial phenotype.

3.2. TGF $\beta_{1}$-Induced Alterations in Epithelial and Mesenchymal Markers and Markers of Apoptosis In Vivo. Using a $\mathrm{TGF} \beta_{1}$ transgenic mouse system, where active TGF $\beta_{1}$ is overexpressed specifically in an inducible manner in the lung epithelium, we measured elevated soluble collagen in the lungs of mice, after day 10 of transgene activation, which showed a significant increase starting at day 10 of transgene activation and continued to increase to day 21 (Figure 3(a)). To assess any changes in epithelial and mesenchymal phenotype, we also assessed the gene expression levels of a panel of genes in whole lung RNA. Here, alterations in a number of genes was apparent as early as day 3, with increases in collagen Ia1, fibronectin, WISP, and CTGF being observed (Figure 3(b)). At days 7 and 10, mesenchymal gene expression, although upregulated compared to matching wild-type control mice, began to decrease compared to day 

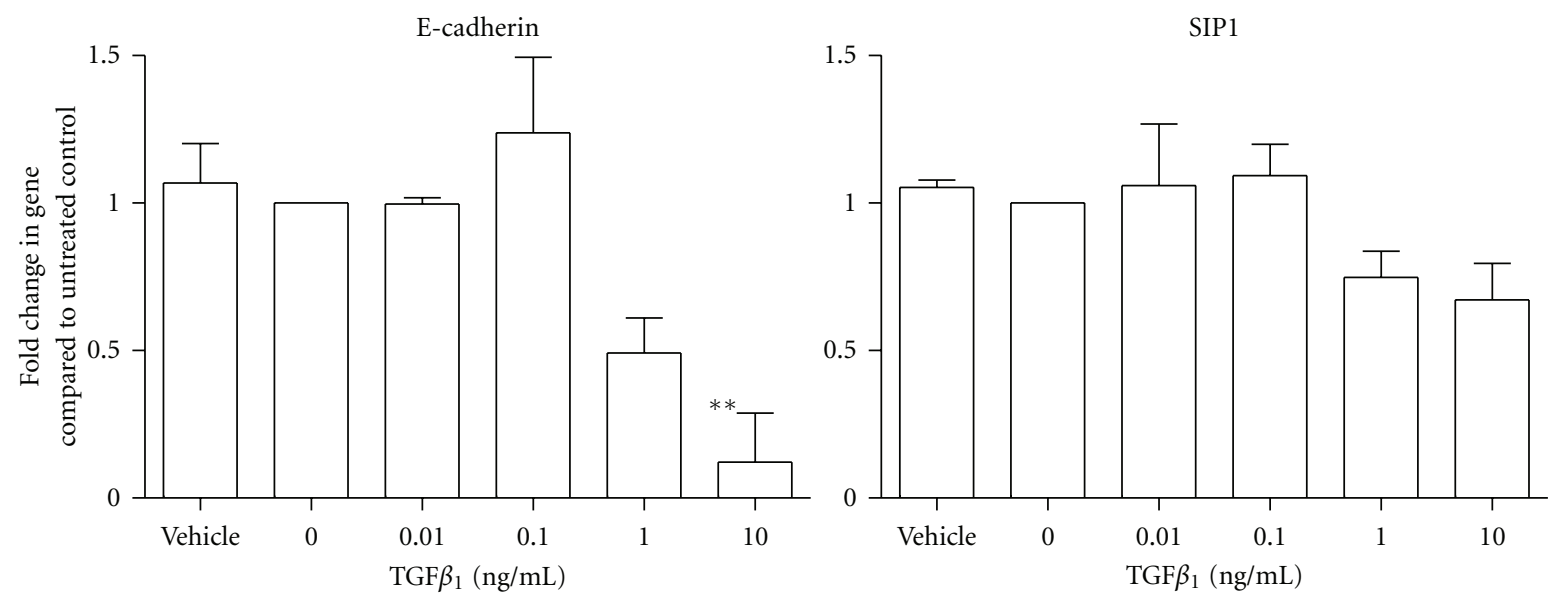

(a)
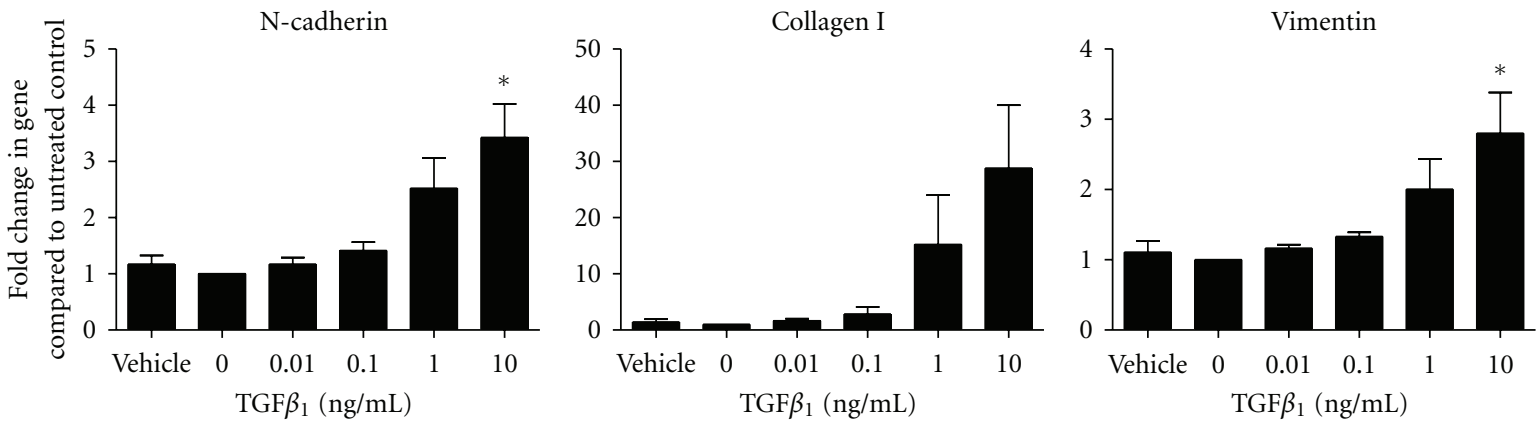

(b)
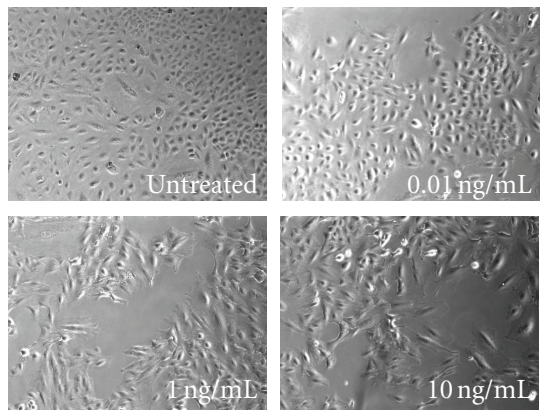

(c)
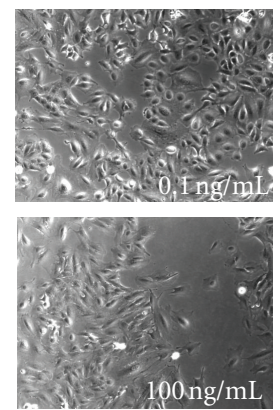

Fer. $100 \mathrm{ng} / \mathrm{mL}$
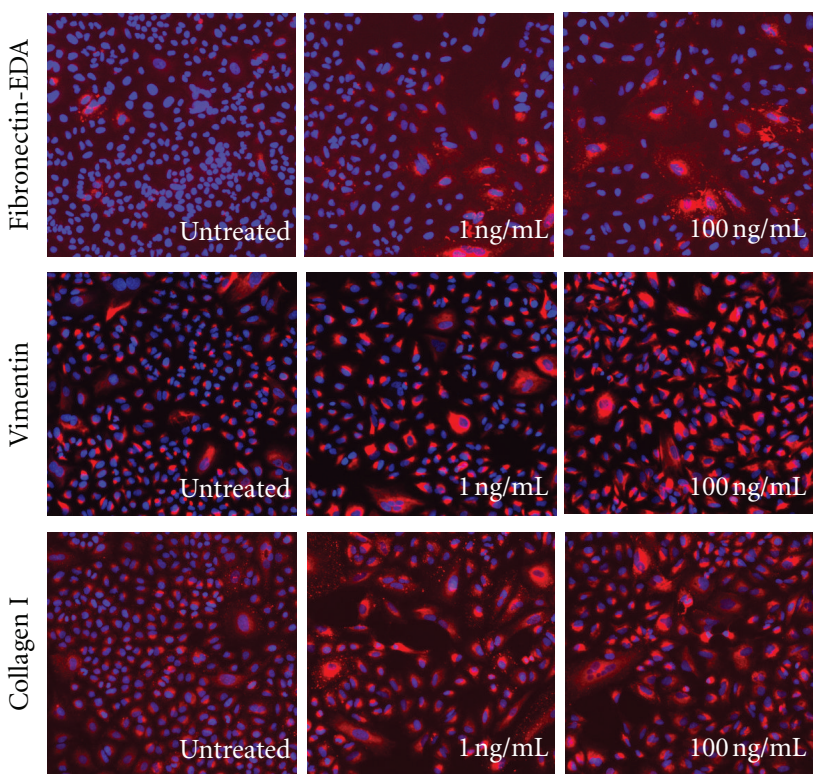

(d)

Figure 1: TGF $\beta_{1}$ induced EMT in A549 cells. Epithelial (a) and mesenchymal (b) gene expression in A549 cells stimulated with TGF $\beta_{1}$ $(0.01,0.1,1,10$, and $100 \mathrm{ng} / \mathrm{mL})$ vehicle control or media alone for 24 hours. Gene expression levels were determined using QuantiGene 2.0 branched DNA technology. Data was analysed relative to the expression of the endogenous control (PPIB) and normalised to unstimulated cells. Data shown represents mean \pm SEM of three independent experiments $(n=3)$. A549 cell morphology changes following TGF $\beta_{1}$ $(0.01,0.1,1,10$, and $100 \mathrm{ng} / \mathrm{mL})$ or media alone treatment for 72 hours, live cells were visualised using phase contrast microscopy, and representative images are shown (c). Immunocytochemistry of the mesenchymal markers; fibronectin-EDA, vimentin and collagen I (red), and nuclei (blue) in A549 cells treated with TGF $\beta_{1}$ ( 1 and $100 \mathrm{ng} / \mathrm{mL}$ ) or media alone for 72 hours. Cells were visualised using an ArrayScan (Cellomics), and representative images are shown (d). ${ }^{*} P<0.05,{ }^{* *} P<0.01$ statistically significant versus vehicle control, using a one-way ANOVA with Dunnett's posttest. 


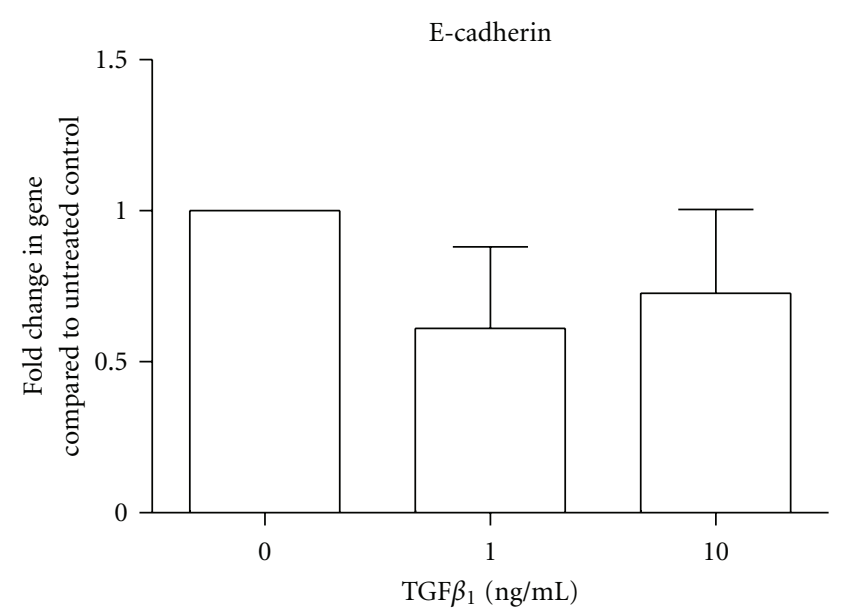

(a)
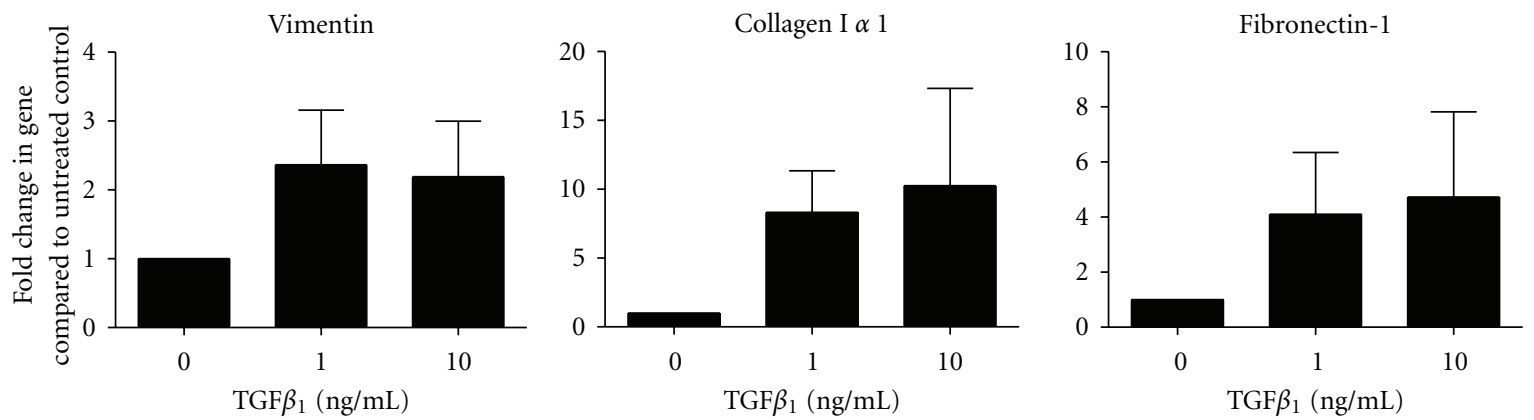

(b)
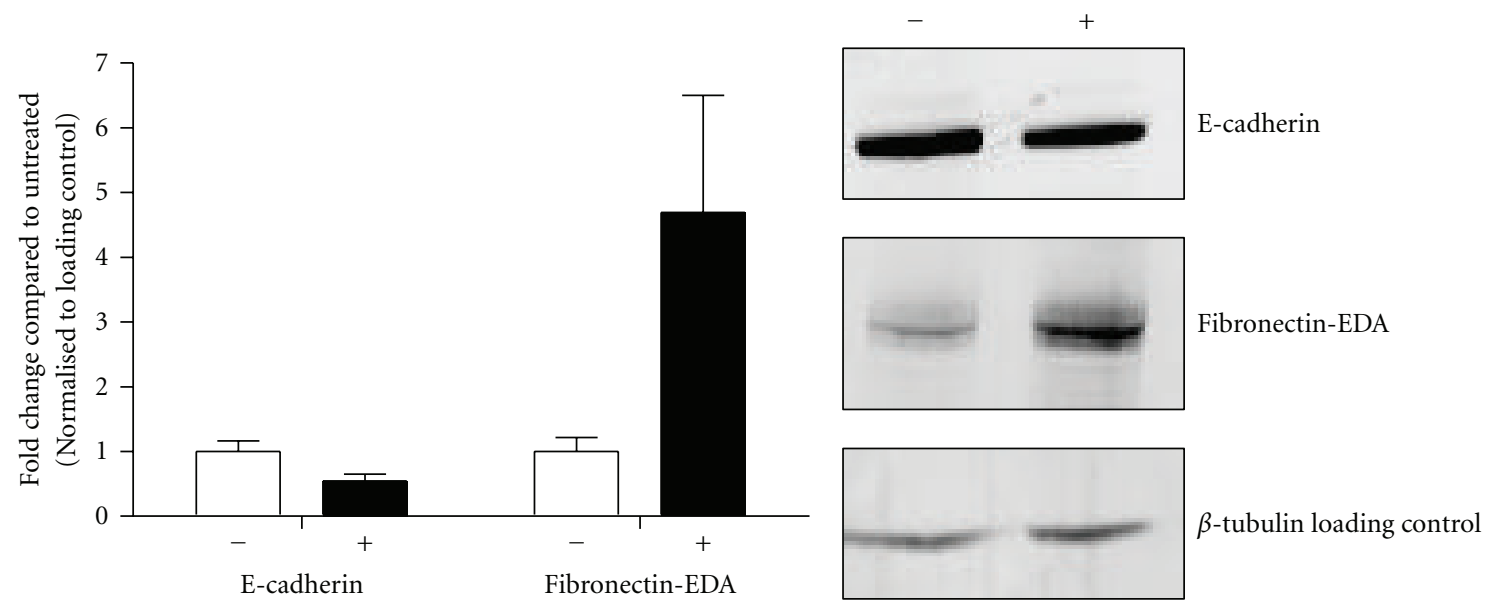

(c)

(d)

FIGURE 2: TGF $\beta_{1}$ induced EMT in NHBE cells. Epithelial (a) and mesenchymal (b) gene expression in NHBE cells stimulated with TGF $\beta_{1}$ (1 and $10 \mathrm{ng} / \mathrm{mL}$ ) or media alone for 24 hours. Gene expression levels were determined using QuantiGene 2.0 branched DNA technology. Data was analysed relative to the expression of the endogenous control (PPIB) and normalised to unstimulated cells. Data shown represents mean \pm SEM of at least three independent experiments $(n=3)$. (c) Effects of TGF $\beta_{1}$-induced EMT on E-cadherin and fibronectin-1 protein levels assessed by western blot and quantified using densitometry. Data shown represents mean \pm SEM of at least three independent experiments $(n=3)$. (d) Representative western blot evaluation of NHBE cells treated with $10 \mathrm{ng} / \mathrm{mL}$ of TGF $\beta_{1}(+)$ or media alone $(-)$ was performed after 48 hours to determine levels of E-cadherin and fibronectin-1. $\beta$-tubulin loading control was used. Data is shown from one experiment. 


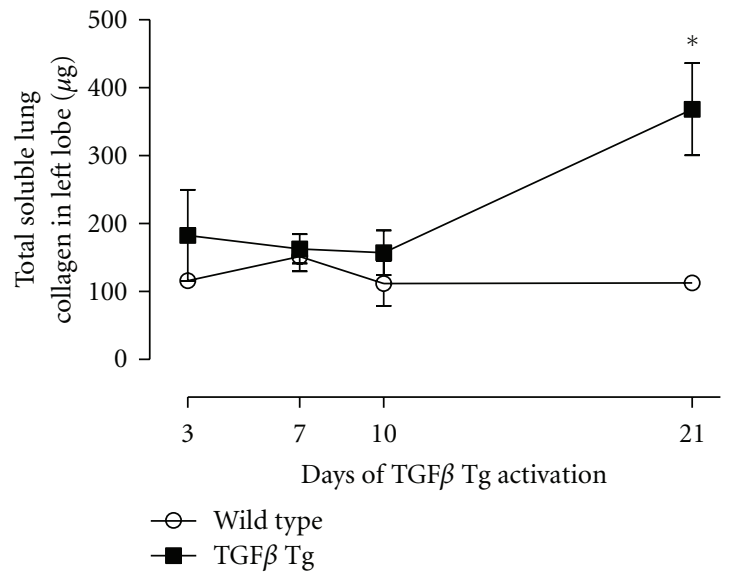

(a)

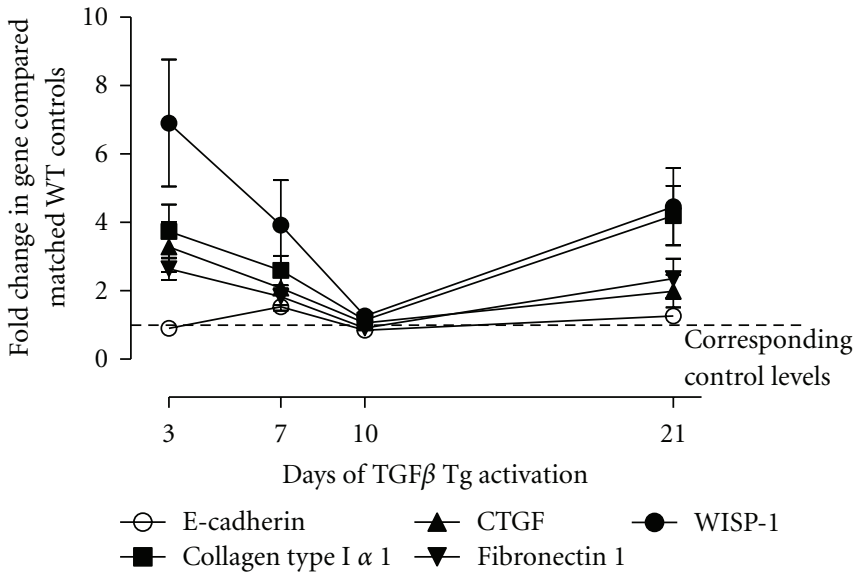

(b)

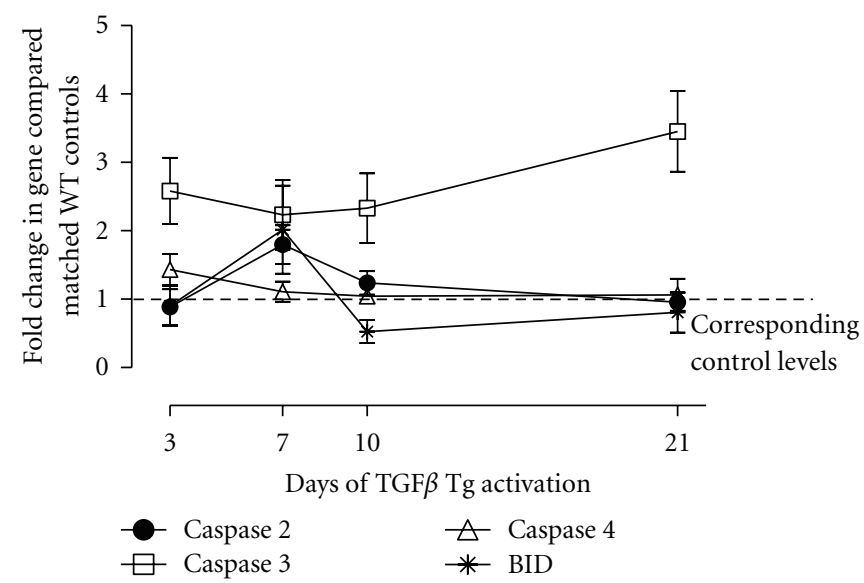

(c)

FIGURE 3: Evidence of increased collagen deposition and changes in epithelial, mesenchymal, and apoptotic gene expression following lungspecific TGF $\beta_{1}$ transgene overexpression. TGF $\beta_{1}$ was overexpressed by introducing doxycycline $(0.5 \mathrm{mg} / \mathrm{mL})$ into the animals water; wildtype controls also received doxycycline for 3, 7, 10, and 21 days. Total soluble collagen was measured in homogenates from the left lobe of the lung using the Sircol assay (a). RNA was isolated from whole lung and qRT-PCR was performed to identify EMT (b) and proapoptotic (c) regulated genes. Fold change in gene expression was calculated by comparing TGF $\beta_{1}$ mice with wild-type mice at matching time points. Data represent mean \pm SEM of at least $n=3$ mice at each time point. ${ }^{*} P<0.05$ using Student's $t$-test.

3. However, there was a subsequent increase again at day 21 , suggesting a bimodal TGF $\beta_{1}$-mediated response. The epithelial marker, E-cadherin, appeared to be only slightly downregulated at day 3 (0.9-fold) and day 10 (0.9-fold) and slightly increased at day 7 (1.5-fold) and day 21 (1.3-fold).

High levels of TGF $\beta_{1}$ are known to promote apoptosis in vitro [25]. Moreover, inhibiting apoptosis with a pancaspase inhibitor Z-VAD reduced subsequent fibrosis [18]. Therefore, we next determined the relative expression of apoptotic markers present in the lungs of TGF $\beta_{1}$ transgenic mice (Figure 3(c)). TGF $\beta_{1}$ induced expression of caspase-3 (2.6-fold) at the early day 3 time point, although this then dropped slightly at days 7 and 10 but by day 21 this had increased further to 3.5 -fold. BID and caspase-2 showed a peak in mRNA expression at day 7 of 2.0 - and 1.8 -fold, respectively, compared to all other time points. Caspase- 4 showed minimal changes peaking at day 3 (1.4-fold) but then gradually decreased.
3.3. Increased Collagen Deposition Is Associated with Epithelial, Mesenchymal, and Apoptotic Changes in the Bleomycin Model of Lung Fibrosis. We next profiled the expression of epithelial and mesenchymal markers, along with apoptosis markers during a bleomycin-induced model of lung fibrosis. To confirm the extent of lung fibrosis, we determined the levels of lung collagen using a hydroxyproline assay. Here, at days 7 and 14, significant levels of collagen were detected compared to saline controls (Figure 4(a)). Assessment of epithelial and mesenchymal markers at these time points indicates an increase in mesenchymal marker upregulation at day 3; however, this was further increased at day 7 with increased expression of collagen Ia1 (3.9-fold), CTGF (4.0fold), fibronectin-1 (2.7-fold), and WISP-1 (5.5-fold). At day 14, collagen Ial and WISP-1 further increased to 7.8and 6.8-fold, respectively, whereas CTGF and fibronectin remained relatively unchanged. In contrast, the epithelial marker E-cadherin appeared to be downregulated at day 3 


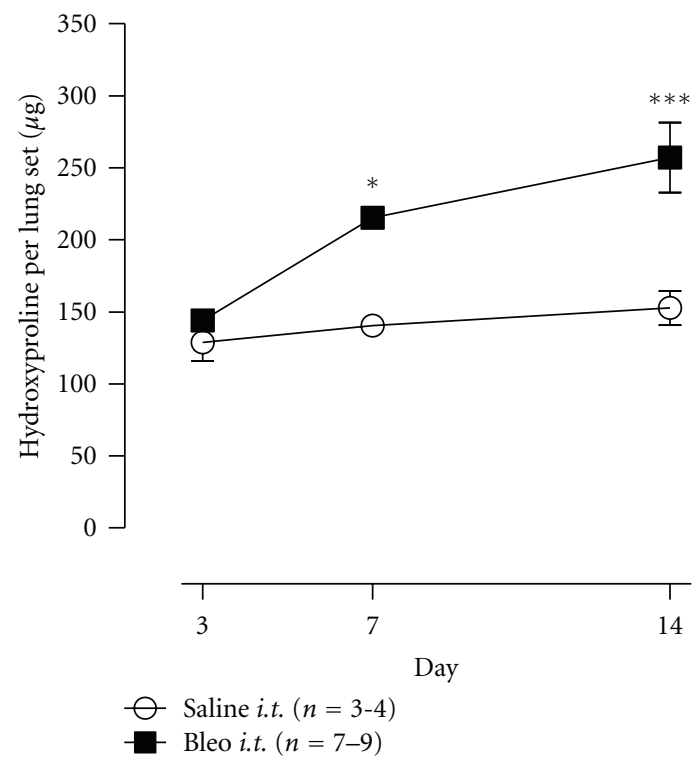

(a)

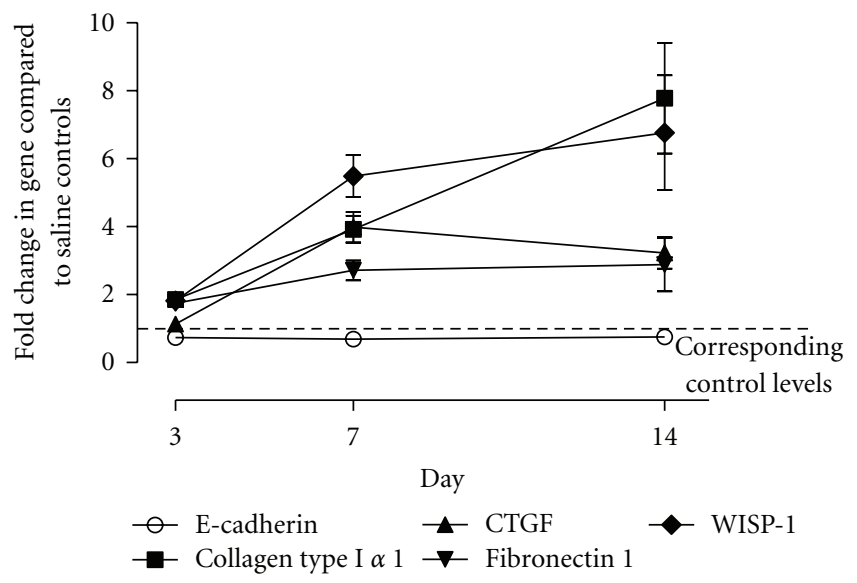

(b)

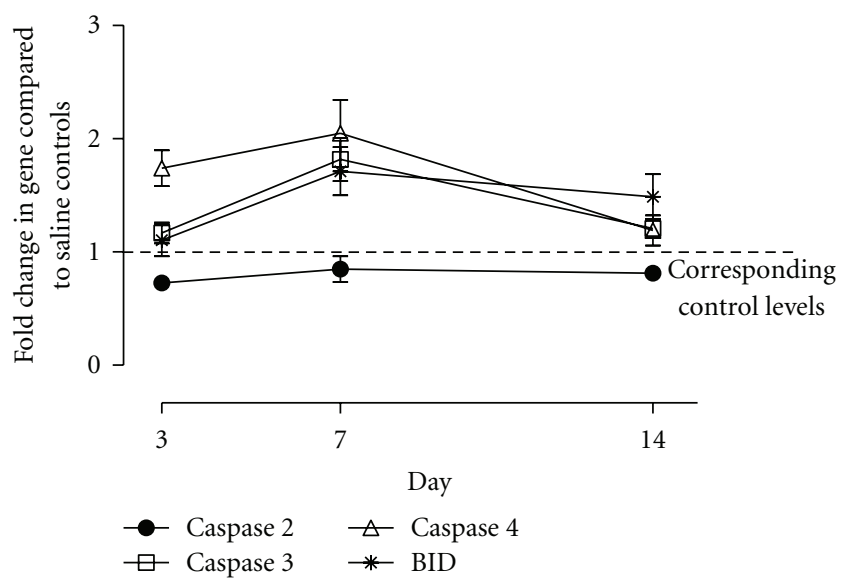

(c)

FIGURE 4: Evidence of increased collagen deposition and changes in epithelial, mesenchymal, and apoptotic gene expression in bleomycintreated mice. Mice were given bleomycin sulphate $(0.015 \mathrm{U} / 30 \mu \mathrm{L} /$ mouse $)$ or saline i.t. at day 0 and then at days 3,7 , and 14 , lungs were removed for subsequent analysis. Lung collagen was measured using hydroxyproline assay in homogenates from whole lung (a). RNA was isolated from whole lung and qRT-PCR was performed to identify EMT (b) and proapoptotic (c) regulated genes. Data points represent mean \pm SEM of at least $n=3$ mice at each time point. ${ }^{*} P<0.05$, ${ }^{* * *} P<0.005$ using Student's $t$-test.

(0.74-fold), day 7 (0.70-fold), and day 14 (0.75-fold) and this moderate downregulation remained relatively unchanged across the duration of the time course.

We also determined changes in apoptotic markers across the bleomycin-induced model (Figure 4(c)). Here we measured no change in caspase-2 gene expression; however, caspase-3, caspase-4, and BID all showed increased mRNA expression peaking for all of the genes at day 7 (1.8, 2.0, and 1.7 , resp.).

3.4. Alterations in Epithelial, Mesenchymal and Apoptotic Markers in the Lungs of IPF Patients. To determine if any of the gene changes observed in the in vivo models correlated with human disease, we next assessed the relative levels of epithelial and mesenchcymal markers in surgical lung biopsies from IPF patients and compared them to normal controls. The IPF patient population was further subdivided into slow and rapid progressors as previously described [26]. We noticed a decrease in the epithelial markers keratin 18 and keratin 19 in both slow and rapid progressors (Figure 5(a)). In contrast, the mesenchymal markers collagen Ia2, collagen IIIa1, and vimentin were all increased in both cohorts of IPF patients (Figure 5(b)). Interestingly, in rapid progressors, the level of mesenchymal marker expression was higher than that of the slower progressors whereas the opposite was true for keratin 18 and keratin 19, with slow progressors showing decreased expression in comparison to rapid progressors. In addition, we measured the apoptotic marker, GSK3 $\beta$, which was increased in IPF patients and again more profound in the 

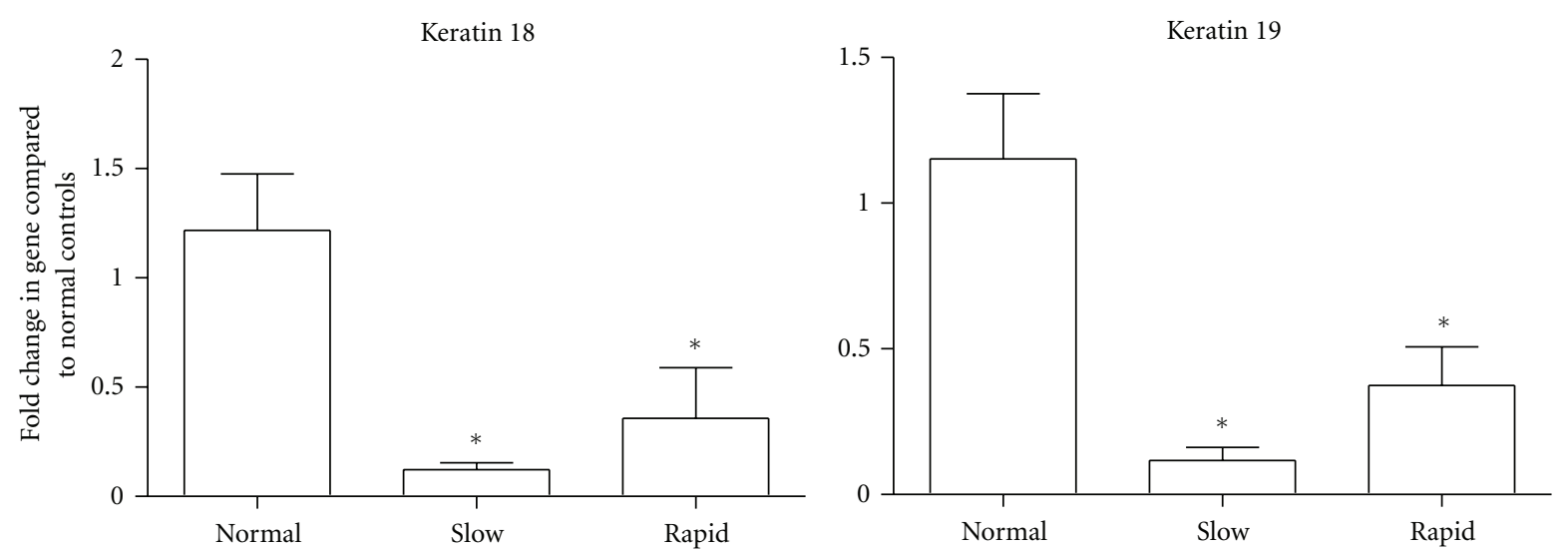

(a)
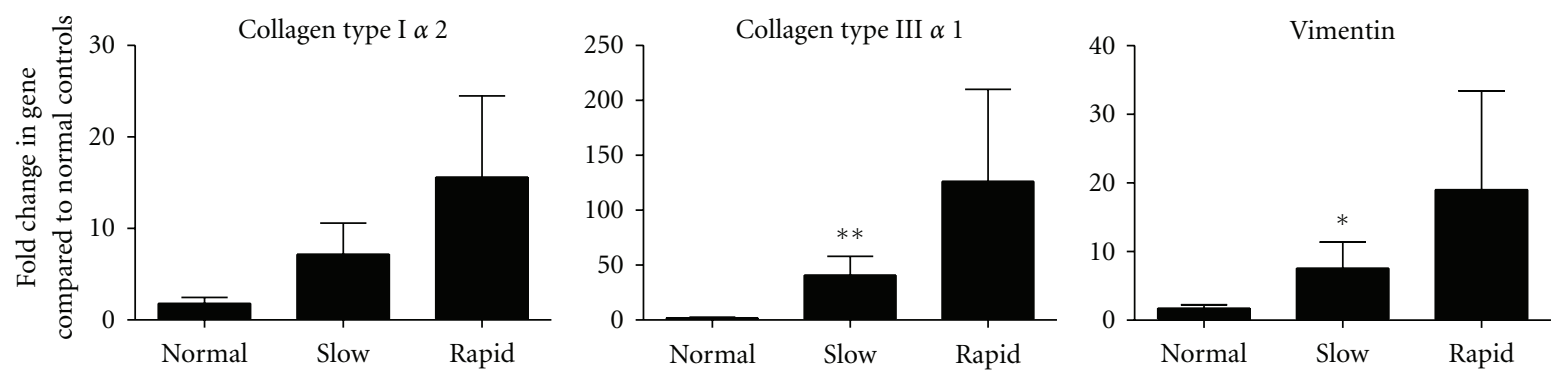

(b)

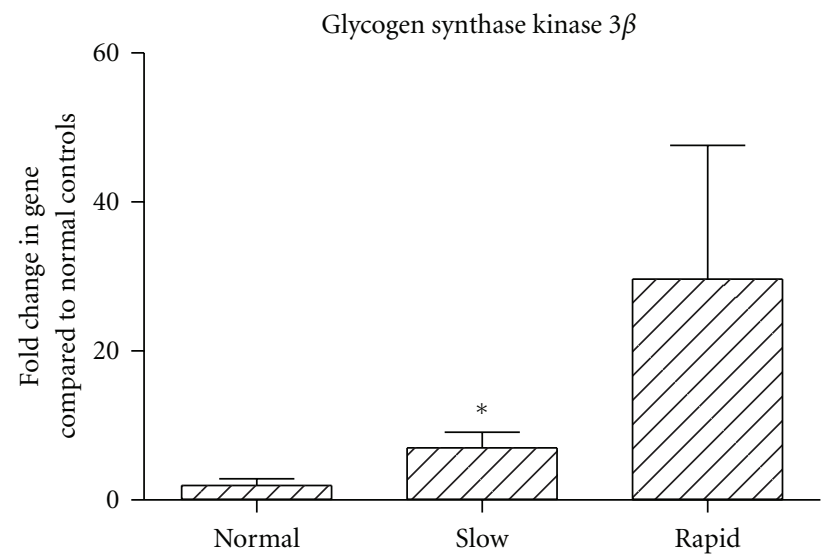

(c)

FIGURE 5: Changes in mesenchymal, epithelial, and apoptotic markers in IPF patients. RNA was isolated from surgical lung biopsies of IPF patients and qRT-PCR was performed to determine the expression of epithelial (a), mesenchymal (b), and apoptotic (c) regulated genes in normal, slow, and rapid progressors. Data points represent mean \pm SEM of $n=6-10$ for each group. ${ }^{*} P<0.05$, ${ }^{* *} P<0.01$ significance versus normal control using Student's $t$-test.

rapid-progressing cohort. Overall this disease-relevant data supports epithelial, mesenchymal, and apoptotic alterations with the progression of IPF.

\section{Discussion}

The aims of this study were to profile epithelial and mesenchymal changes during in vitro and in vivo models of lung fibrosis and associate this with clinical disease. We initially assessed the in vitro effects of TGF $\beta_{1}$ on lung epithelial cells.
We then assessed the epithelial changes mediated by TGF $\beta_{1}$ overexpression directly in the lungs of mice and compared this with the bleomycin-induced lung fibrosis model of IPF. Finally, we measured markers of epithelial change in lung biopsies from rapidly progressive and slowly progressive IPF patients.

EMT itself is an essential mechanism which plays a role in embryonic development and normal wound healing; however, settings where EMT is dysregulated can lead to cancer metastases and organ fibrosis [27]. EMT is a dynamic process whereby polarised epithelial cells undergo 
a morphological change to differentiated mesenchymal cells which are more invasive, motile, and resistant to apoptosis $[28,29]$. During EMT, epithelial and tight junction proteins, including E-cadherin and certain cytokeratins, are lost; in contrast mesenchymal markers such as vimentin, $\mathrm{N}$ cadherin, connective tissue growth factor (CTGF), wnt inducible signalling protein-1 (WISP-1), and fibronectin are gained $[12,30,31]$. Due to this change in multiple and often widely expressed markers, a panel or signature of changes in EMT markers are normally assessed [12].

Whether EMT exists in IPF is a controversial research area [32]. Primary epithelial cells isolated from the lungs of asthmatics are more prone to undergo EMT ex vivo [33]. In IPF, there is some evidence that isolated alveolar epithelial cells from patients express increased mesenchymal proteins and TGF $\beta_{1}$ regulates this process [34]. However, due to the practical limitations associated with isolating epithelial cells from IPF patients [35], it is difficult to profile EMT in disease-relevant epithelial cells. Therefore we assessed A549 and NHBE in our EMT conditions. These cells are not derived from the main site of IPF fibrosis, the parenchyma. However, they do provide a useful tool in assessing epithelial biology in vitro. In this system, A549 cells were more sensitive to EMT than NHBE cells, which may be explained by the source of A549 being from a lung tumor and EMT is known to be a key feature of cancer progression [29].

In the primary NHBE cells, minimal morphological changes were observed in response to TGF $\beta_{1}$, nor was there a significant decrease of epithelial marker expression. However, there was a dose-dependent increase in mesenchymal marker expression. The minimal changes in Ecadherin, a tight junction protein, unsurprisingly correlated with the lack of alteration of morphology. There are other reports demonstrating TGF $\beta_{1}$-induced EMT in primary NHBE cells; however, one publication fails to show or comment on any morphological changes occurring [36] and the second report, although shows some morphological change, this only occurs after 5 days of TGF $\beta_{1}$ stimulation [37]. Therefore, the lack of epithelial changes in NHBEs in our system may be due to donor variability or differing aging/passaging of the cells. The cells used in our study were from a nonsmoker; this information was not provided in the above reports which could also modify the response of the cells. Future studies could assess the effects of aging and donor variability on TGF $\beta_{1}$-induced EMT responses. Another potential reason as to why the NHBEs were not as prone to losing epithelial phenotype is that they are derived from healthy donor lung tissue.

The lungs of IPF patients contain numerous cell types that exhibit altered phenotype and altered responsiveness to growth factor stimulation. Therefore, we used in vivo models to assess these phenomena in a more physiologically relevant, multicellular setting. An acute response in the TGF $\beta_{1}$ transgenic model was shown by increased mesenchymal gene expression at day 3. However, this increase declined and normalised at day 10 and then further increased by day 21. In addition, the epithelial marker E-cadherin remained relatively stable throughout the model but did show a slight increase at days 7 and 21 compared to days 3 and 10. At the protein level, the TGF $\beta_{1}$ transgenic model demonstrated the most significant increases in collagen deposition at day 21 . We hypothesize that at the earlier timepoints, the changes in the epithelial and mesenchymal markers at day 3 and day 7 are more indicative of epithelial injury, which is corroborated with the increased expression of the apoptosis markers at day 3 and day 7. Moreover, inhibition of the early apoptosis in this model attenuates fibrosis [38]; therefore this initial epithelial injury response may be the initiating factor for fibrotic responses that occur after day 10. Further assessment of the apparent bimodal effects on fibrosis markers following TGF $\beta_{1}$ Tg overexpression may also determine key changes in TGF $\beta_{1}$ responses as the fibrotic milieu becomes more chronic.

The bleomycin-induced lung fibrosis model is a model commonly used to assess pathways and features of IPF. There are significant differences between this model and clinical IPF, such as the spontaneous resolution of fibrosis in mice. However, key hallmarks observed in the clinical setting also occur following intratracheal bleomycin administration, namely, epithelial apoptosis and the presence of fibroblastic foci. In this study, we saw a gradual increase in mesenchymal associated gene expression across the time course. Fibronectin-1 and CTGF appeared to somewhat stabilise at day 7, whereas collagen Ial and WISP-1 continued to increase at day 14. E-cadherin remained relatively unchanged across the time course; although there was a minimal decrease in expression, this reduction may have been diluted due to whole lung tissue RNA analyzed, rather than specific epithelial regions. Subsequent studies may therefore confirm that EMT is occurring in this model. Furthermore, we noted an increase in the apoptotic markers caspase-3, caspase4 , and BID which peaked at day 7. In previous studies it has also been identified that bleomycin-induced mice show evidence of bronchial epithelial cell injury by TUNEL staining [39]; however there are no known references, to our knowledge, where blocking apoptosis through caspase inhibition modulates lung fibrosis. Interestingly, it has already been suggested that apoptosis at the advancing edges of wounds is essential for the normal wound-healing response [40]. Moreover, based on the kinetics of the apoptosis genes and the epithelial and mesenchymal gene changes, the TGF $\beta_{1}$ transgenic model may have an initial wound-healing response which is not associated with EMT. Moreover, this wound-healing response subsides at later stages. For all gene analyses, whole lung RNA was used. In future studies immunohistochemical analysis of tissue sections or laser capture microdissection will help to determine any discreet epithelial changes in either model.

In the final part of this study we evaluated epithelial and mesenchymal markers and apoptotic gene expression changes in lung samples from slow- and rapid-progressing IPF patients. Here we also saw an increase in mesenchymal markers, but we also detected a reduction in the epithelial markers, KRT18 and KRT19, when comparing IPF to healthy control donor lung. In addition, these alterations in epithelial-mesenchymal gene expression levels were also associated with a robust increase in the apoptotic marker GSK $3 \beta$, which was also more profound in rapid-progressing 
IPF patients. This data collectively suggests that markers of epithelial and mesenchymal cells are altered and associated with the degree of apoptosis in IPF patients compared to normal's. Moreover, both mechanisms are more profound in the rapidly progressing IPF patient population, suggesting EMT is present, but this is more apparent in the rapid patients versus the slow-progressing patients.

One of the factors associated with IPF is repeated epithelial injury; however the hypothesis that EMT is driving IPF is controversial. The initial defining study describing EMT in IPF demonstrated a concomitant expression of epithelial and mesenchymal marker expression in lung sections [12]. However, whether this is a bystander phenomenon or actually promoting disease severity is unclear. In this study we describe experimental settings where we have shown that this phenomenon exists, namely, through TGF $\beta_{1}$ promoting alterations in epithelial and mesenchymal cell phenotype. However, these alterations may simply be markers of epithelial injury and apoptosis due to the level of stimulus that was used. Moreover, there is increased lung cancer in patients with IPF [41]; therefore it would be important to exclude these patients from analyses determining the presence of EMT in IPF. Furthermore, assessing therapeutic agents that specifically and uniquely block EMT in experimental models will define whether this cell differentiation pathway contributes to lung fibrosis development.

\section{Conflict of Interests}

This is to confirm that no one of the authors have direct or indirect financial relation with the commercial identities mentioned above.

\section{References}

[1] V. Tzilas, A. Koti, D. Papandrinopoulou, and G. Tsoukalas, "Prognostic factors in idiopathic pulmonary fibrosis," American Journal of the Medical Sciences, vol. 338, no. 6, pp. 481-485, 2009.

[2] V. Navaratnam, K. M. Fleming, J. West et al., "The rising incidence of idiopathic pulmonary fibrosis in the UK," Thorax, vol. 66, no. 6, pp. 462-467, 2011.

[3] S. K. Frankel and M. I. Schwarz, "Update in idiopathic pulmonary fibrosis," Current Opinion in Pulmonary Medicine, vol. 15, no. 5, pp. 463-469, 2009.

[4] T. J. Gross and G. W. Hunninghake, "Idiopathic pulmonary fibrosis," The New England Journal of Medicine, vol. 345, no. 7, pp. 517-525, 2001.

[5] P. W. Noble and R. J. Homer, "Idiopathic pulmonary fibrosis: new insights into pathogenesis," Clinics in Chest Medicine, vol. 25, no. 4, pp. 749-758, 2004.

[6] G. Raghu and J. Chang, "Idiopathic pulmonary fibrosis: current trends in management," Clinics in Chest Medicine, vol. 25, no. 4, pp. 621-636, 2004.

[7] M. Selman, T. E. King, and A. Pardo, "Idiopathic pulmonary fibrosis: prevailing and evolving hypotheses about its pathogenesis and implications for therapy," Annals of Internal Medicine, vol. 134, no. 2, pp. 136-151, 2001.
[8] K. Kuwano, "Involvement of epithelial cell apoptosis in interstitial lung diseases," Internal Medicine, vol. 47, no. 5, pp. 345$353,2008$.

[9] R. J. Homer, J. A. Elias, C. G. Lee, and E. Herzog, "Modern concepts on the role of inflammation in pulmonary fibrosis," Archives of Pathology and Laboratory Medicine, vol. 135, no. 6, pp. 780-788, 2011.

[10] B. C. Willis, J. M. Liebler, K. Luby-Phelps et al., "Induction of epithelial-mesenchymal transition in alveolar epithelial cells by transforming growth factor- $\beta 1$ : potential role in idiopathic pulmonary fibrosis," American Journal of Pathology, vol. 166, no. 5, pp. 1321-1332, 2005.

[11] K. K. Kim, M. C. Kugler, P. J. Wolters et al., "Alveolar epithelial cell mesenchymal transition develops in vivo during pulmonary fibrosis and is regulated by the extracellular matrix," The Proceedings of the National Academy of Sciences of the United States of America, vol. 103, no. 35, pp. 13180-13185, 2006.

[12] B. C. Willis and Z. Borok, "TGF- $\beta$-induced EMT: mechanisms and implications for fibrotic lung disease," American Journal of Physiology_Lung Cellular and Molecular Physiology, vol. 293, no. 3, pp. L525-L534, 2007.

[13] N. J. Lomas, K. L. Watts, K. M. Akram, N. R. Forsyth, and M. A. Spiteri, "Idiopathic pulmonary fibrosis: immunohistochemical analysis provides fresh insights into lung tissue remodelling with implications for novel prognostic markers," International Journal of Clinical and Experimental Pathology, vol. 5, no. 1, pp. 58-71, 2012.

[14] S. Grünert, M. Jechlinger, and H. Beug, "Diverse cellular and molecular mechanisms contribute to epithelial plasticity and metastasis," Nature Reviews Molecular Cell Biology, vol. 4, no. 8, pp. 657-665, 2003.

[15] M. Iwano, D. Plieth, T. M. Danoff, C. Xue, H. Okada, and E. G. Neilson, "Evidence that fibroblasts derive from epithelium during tissue fibrosis," Journal of Clinical Investigation, vol. 110, no. 3, pp. 341-350, 2002.

[16] N. Khalil, R. N. O’Connor, H. W. Unruh et al., "Increased production and immunohistochemical localization of transforming growth factor- $\beta$ in idiopathic pulmonary fibrosis," American Journal of Respiratory Cell and Molecular Biology, vol. 5, no. 2, pp. 155-162, 1991.

[17] N. Khalil, T. V. Parekh, R. O'Connor et al., "Regulation of the effects of TGF- $\beta 1$ by activation of latent TGF- $\beta 1$ and differential expression of TGF- $\beta$ receptors (T $\beta$ R-I and T $\beta$ R-II) in idiopathic pulmonary fibrosis," Thorax, vol. 56, no. 12, pp. 907-915, 2001.

[18] C. G. Lee, S. J. Cho, M. J. Kang et al., "Early growth response gene 1-mediated apoptosis is essential for transforming growth factor $\beta 1$-induced pulmonary fibrosis," Journal of Experimental Medicine, vol. 200, no. 3, pp. 377-389, 2004.

[19] C. M. Swaisgood, E. L. French, C. Noga, R. H. Simon, and V. A. Ploplis, "The development of bleomycin-induced pulmonary fibrosis in mice deficient for components of the fibrinolytic system," American Journal of Pathology, vol. 157, no. 1, pp. 177-187, 2000.

[20] M. D. Burdick, L. A. Murray, M. P. Keane et al., "CXCL11 attenuates bleomycin-induced pulmonary fibrosis via inhibition of vascular remodeling," American Journal of Respiratory and Critical Care Medicine, vol. 171, no. 3, pp. 261-268, 2005.

[21] K. R. Flaherty, T. E. King, G. Raghu et al., "Idiopathic interstitial pneumonia: what is the effect of a multidisciplinary approach to diagnosis?" American Journal of Respiratory and Critical Care Medicine, vol. 170, no. 8, pp. 904-910, 2004. 
[22] Y. Yamauchi, T. Kohyama, H. Takizawa et al., "Tumor necrosis factor- $\alpha$ enhances both epithelial-mesenchymal transition and cell contraction induced in A549 human alveolar epithelial cells by transforming growth factor- $\beta 1$," Experimental Lung Research, vol. 36, no. 1, pp. 12-24, 2010.

[23] H. Kasai, J. T. Allen, R. M. Mason, T. Kamimura, and Z. Zhang, "TGF- $\beta 1$ induces human alveolar epithelial to mesenchymal cell transition (EMT)," Respiratory Research, vol. 6, article 56, 2005.

[24] L. A. Borthwick, A. Gardner, A. de Soyza, D. A. Mann, and A. J. Fisher, "Transforming growth factor- $\beta 1$ (TGF- $\beta 1$ ) driven epithelial to mesenchymal transition (EMT) is accentuated by tumour necrosis factor $\alpha(\mathrm{TNF} \alpha)$ via crosstalk between the SMAD and NF- $\kappa$ B pathways," Cancer Microenvironment, vol. 5, no. 1, pp. 45-57, 2012.

[25] P. M. Siegel and J. Massagué, "Cytostatic and apoptotic actions of TGF- $\beta$ in homeostasis and cancer," Nature Reviews Cancer, vol. 3, no. 11, pp. 807-821, 2003.

[26] S. R. Oak, L. Murray, A. Herath et al., "A micro RNA processing defect in rapidly progressing idiopathic pulmonary fibrosis," PLoS ONE, vol. 6, no. 6, Article ID e21253, 2011.

[27] E. D. Hay, "The mesenchymal cell, its role in the embryo, and the remarkable signaling mechanisms that create it," Developmental Dynamics, vol. 233, no. 3, pp. 706-720, 2005.

[28] R. Kalluri and E. G. Neilson, "Epithelial-mesenchymal transition and its implications for fibrosis," Journal of Clinical Investigation, vol. 112, no. 12, pp. 1776-1784, 2003.

[29] R. Kalluri and R. A. Weinberg, "The basics of epithelialmesenchymal transition," Journal of Clinical Investigation, vol. 119, no. 6, pp. 1420-1428, 2009.

[30] U. Valcourt, M. Kowanetz, H. Niimi, C. H. Heldin, and A. Moustakas, "TGF- $\beta$ and the Smad signaling pathway support transcriptomic reprogramming during epithelialmesenchymal cell transition," Molecular Biology of the Cell, vol. 16, no. 4, pp. 1987-2002, 2005.

[31] M. Königshoff, M. Kramer, N. Balsara et al., "WNT1-inducible signaling protein-1 mediates pulmonary fibrosis in mice and is upregulated in humans with idiopathic pulmonary fibrosis," Journal of Clinical Investigation, vol. 119, no. 4, pp. 772-787, 2009.

[32] J. R. Rock, C. E. Barkauskas, M. J. Cronce et al., "Multiple stromal populations contribute to pulmonary fibrosis without evidence for epithelial to mesenchymal transition," The Proceedings of the National Academy of Sciences of the United States of America, vol. 108, no. 52, pp. E1475-E1483, 2012.

[33] T. L. Hackett, S. M. Warner, D. Stefanowicz et al., "Induction of epithelial-mesenchymal transition in primary airway epithelial cells from patients with asthma by transforming growth factor- $\beta 1$," American Journal of Respiratory and Critical Care Medicine, vol. 180, no. 2, pp. 122-133, 2009.

[34] C. Marmai, R. E. Sutherland, K. K. Kim et al., "Alveolar epithelial cells express mesenchymal proteins in patients with idiopathic pulmonary fibrosis," American Journal of Physiology-Lung Cellular and Molecular Physiology, vol. 301, no. 1, pp. L71-L78, 2011.

[35] G. Jenkins, A. Blanchard, Z. Borok et al., "In search of the fibrotic epithelial cell: opportunities for a collaborative network," Thorax, vol. 67, no. 2, pp. 179-182, 2012.

[36] A. M. Doerner and B. L. Zuraw, "TGF- $\beta 1$ induced epithelial to mesenchymal transition (EMT) in human bronchial epithelial cells is enhanced by IL- $1 \beta$ but not abrogated by corticosteroids," Respiratory Research, vol. 10, article 100, 2009.

[37] J. Câmara and G. Jarai, "Epithelial-mesenchymal transition in primary human bronchial epithelial cells is Smad-dependent and enhanced by fibronectin and TNF- $\alpha$," Fibrogenesis and Tissue Repair, vol. 3, no. 1, article 2, 2010.

[38] H. R. Kang, G. L. Chun, R. J. Homer, and J. A. Elias, "Semaphorin 7A plays a critical role in TGF- $\beta 1$-induced pulmonary fibrosis," Journal of Experimental Medicine, vol. 204, no. 5, pp. 1083-1093, 2007.

[39] V. V. Polosukhin, A. L. Degryse, D. C. Newcomb et al., "Intratracheal bleomycin causes airway remodeling and airflow obstruction in mice," Experimental Lung Research, vol. 38, no. 3, pp. 135-146, 2012.

[40] N. Schuster and K. Krieglstein, "Mechanisms of TGF- $\beta$ mediated apoptosis," Cell and Tissue Research, vol. 307, no. 1, pp. 1-14, 2002.

[41] I. Le Jeune, J. Gribbin, J. West, C. Smith, P. Cullinan, and R. Hubbard, "The incidence of cancer in patients with idiopathic pulmonary fibrosis and sarcoidosis in the UK," Respiratory Medicine, vol. 101, no. 12, pp. 2534-2540, 2007. 


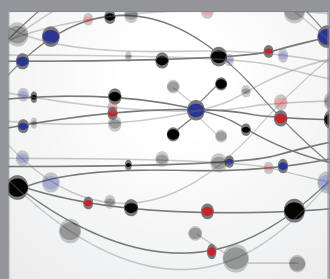

The Scientific World Journal
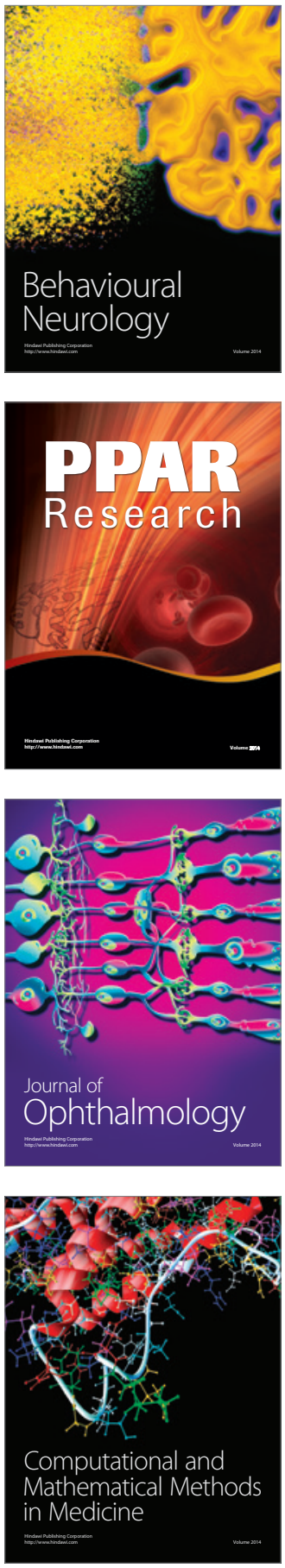

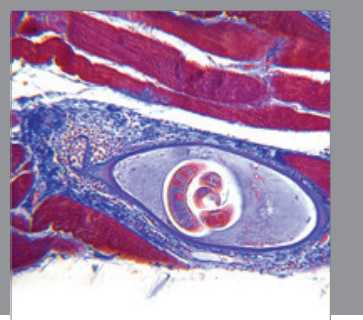

Gastroenterology

Research and Practice
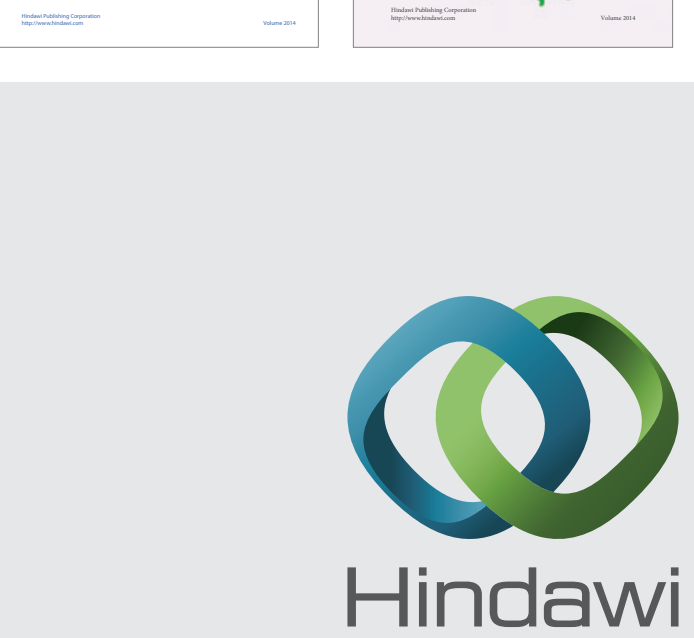

Submit your manuscripts at

http://www.hindawi.com
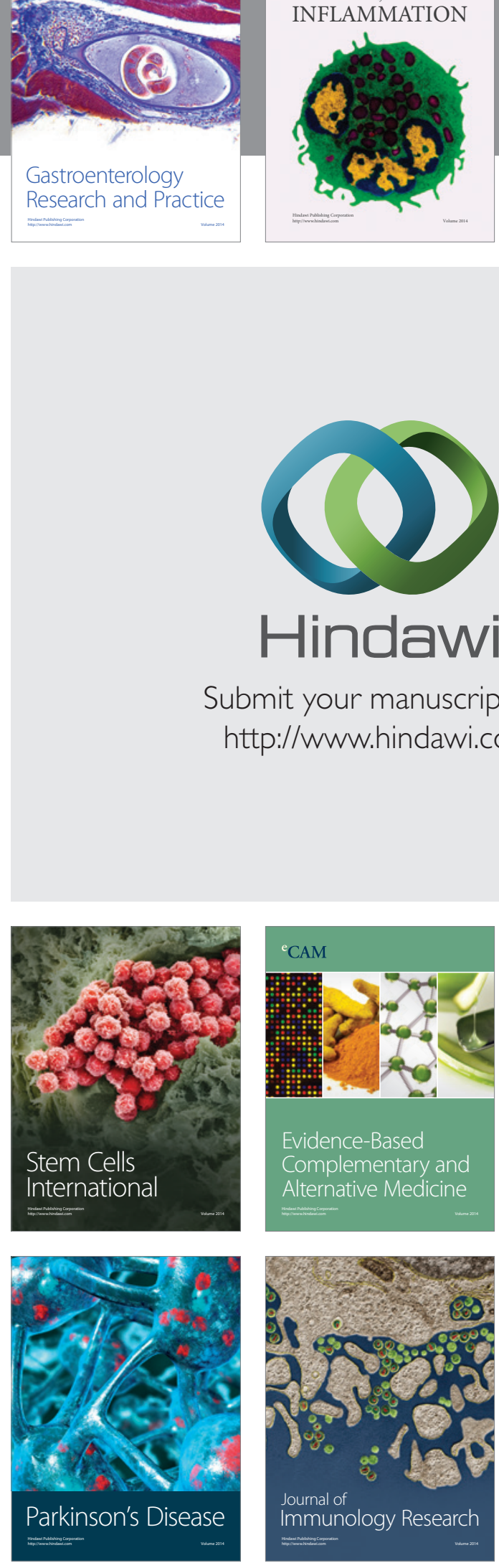

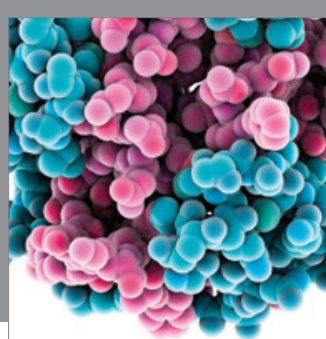

Diabetes Research
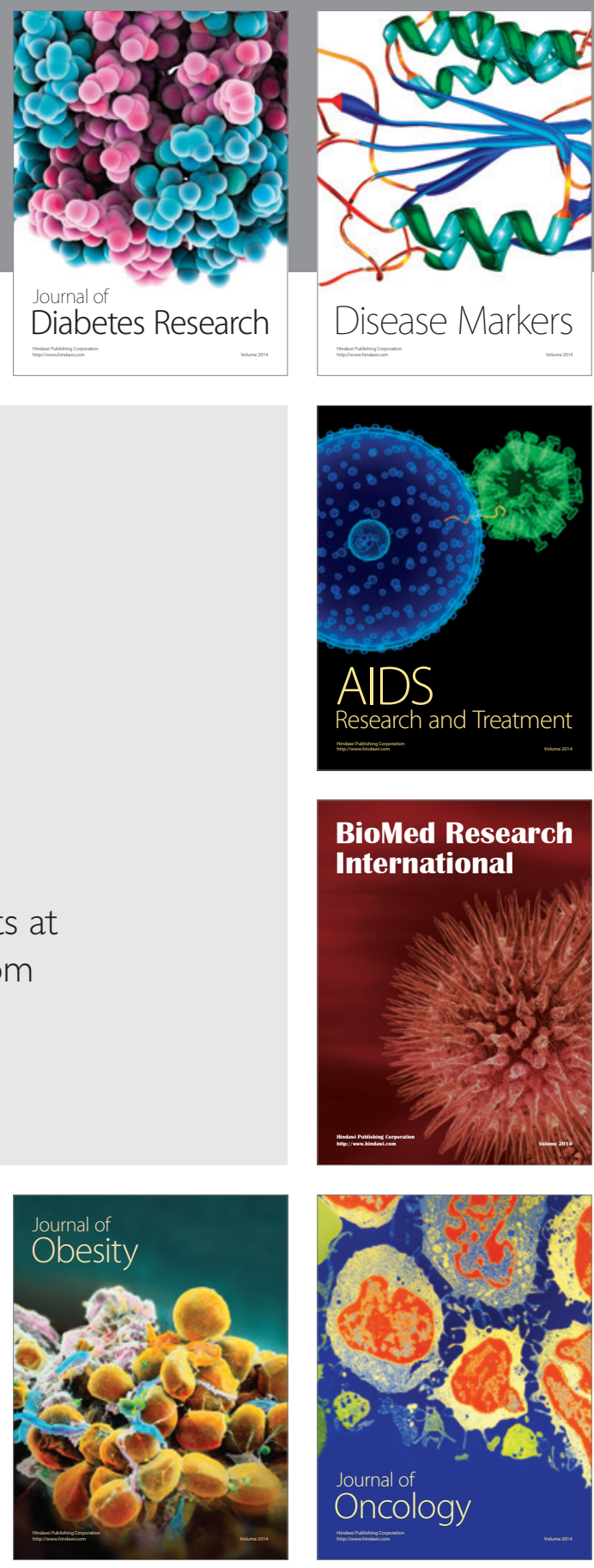

Disease Markers

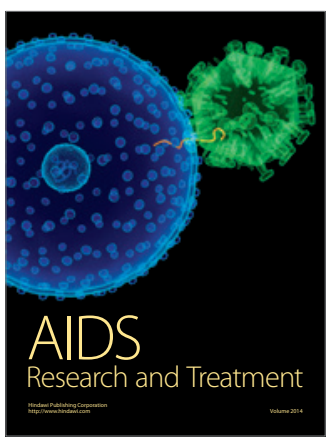

BioMed Research

International
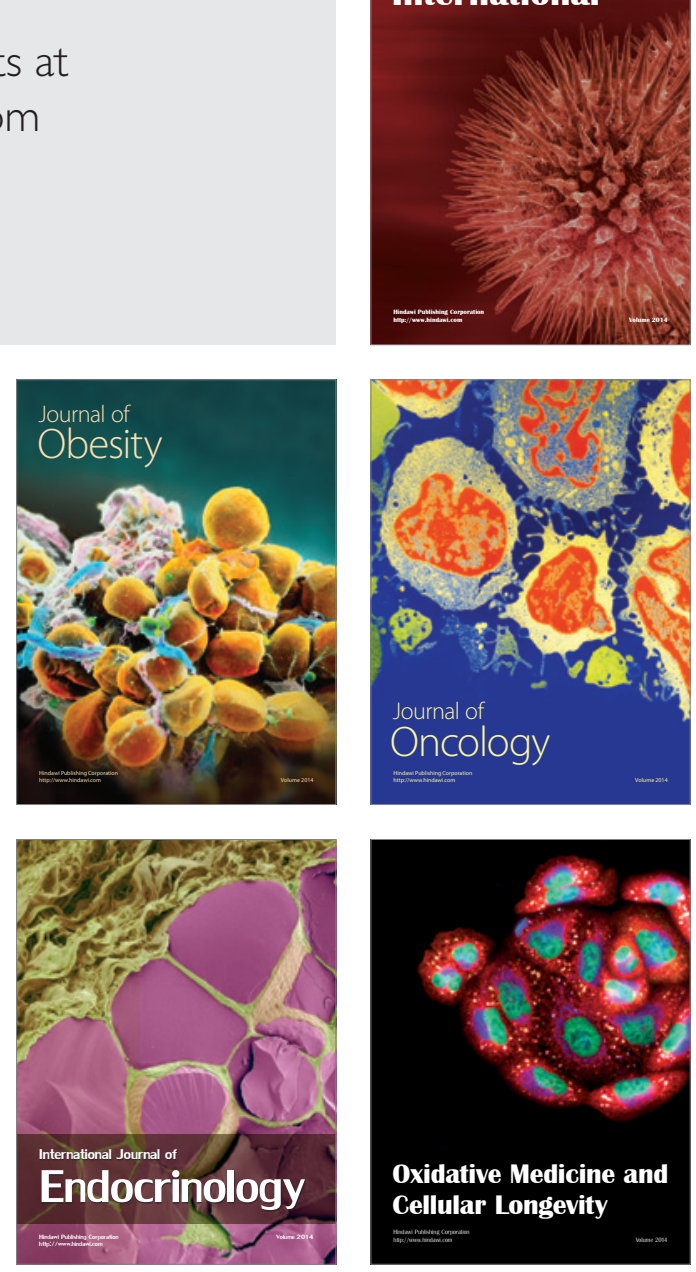